OPEN ACCESS

Edited by:

Giuseppe Spano,

University of Foggia, Italy

Reviewed by:

Emanuele Zannini,

University College Cork, Ireland

Chiara Montanari,

Università degli Studi di Bologna, Italy

${ }^{*}$ Correspondence:

Juliano De Dea Lindner juliano.lindner@ufsc.br

Specialty section:

This article was submitted to

Food Microbiology,

a section of the journal

Frontiers in Microbiology

Received: 30 June 2018 Accepted: 06 August 2018 Published: 21 August 2018

Citation:

Menezes LAA, Minervini F, Filannino $P$,

Sardaro MLS, Gatti M and

De Dea Lindner J (2018) Effects of Sourdough on FODMAPs in Bread and Potential Outcomes on Irritable

Bowel Syndrome Patients

and Healthy Subjects.

Front. Microbiol. 9:1972.

doi: 10.3389/fmicb.2018.01972

\section{Effects of Sourdough on FODMAPs in Bread and Potential Outcomes on Irritable Bowel Syndrome Patients and Healthy Subjects}

\author{
Leidiane A. A. Menezes ${ }^{1}$, Fabio Minervini' ${ }^{2}$ Pasquale Filannino ${ }^{2}$, Maria L. S. Sardaro ${ }^{3}$ \\ Monica Gatti ${ }^{4}$ and Juliano De Dea Lindner ${ }^{1 *}$
}

${ }^{1}$ Department of Food Science and Technology, Federal University of Santa Catarina, Florianópolis, Brazil, ${ }^{2}$ Department of Soil, Plant and Food Sciences, University of Bari Aldo Moro, Bari, Italy, ${ }^{3}$ Department of Human Science and Promotion of the Quality of Life, University of San Raffaele, Rome, Italy, ${ }^{4}$ Department of Food and Drug, University of Parma, Parma, Italy

Background: Fermentable oligosaccharides, disaccharides, monosaccharides and polyols (FODMAPs) are an heterogeneous group of compounds that can be poorly digested and may have a range of effects on gastrointestinal processes. FODMAPs are found in a wide variety of foods, including bread. FODMAPs' intake is associated with the onset of symptoms of irritable bowel syndrome (IBS). On the other hand, some FODMAPs contribute to the healthy maintenance of intestinal microbiota. Volume increase of bread dough commonly relies on the use of two biological leavening agents, sourdough and baker's yeast and, in some cases, a combination of both.

Scope and Approach: The main objective of this review is to discuss the association between FODMAPs and IBS, beneficial effects of FODMAPs on healthy subjects and potential impact of biological leavening agents on FODMAPs content of bread.

Key Findings and Conclusion: Given that yeasts and lactic acid bacteria, the dominant microorganisms in sourdough, may degrade FODMAPs, it would be possible to modulate the FODMAPs concentration in bread, thus positively affecting consumers' health.

\footnotetext{
Keywords: fermentable oligosaccharides, disaccharides, monosaccharides and polyols, irritable bowel syndrome, bread, lactic acid bacteria, yeasts, sourdough
}

\section{INTRODUCTION}

Nowadays, tailored nutritional recommendations may be designed in order to treat or prevent diseases (Betts and Gonzalez, 2016). For instance, lactose- and gluten-containing foods must be avoided by subjects suffering from hypolactasia and celiac disease (De Toro-Martín et al., 2017). Fermentable oligosaccharides, disaccharides, monosaccharides and polyols (FODMAPs) are an heterogeneous group of compounds (most of which are short-chain carbohydrates) that can be poorly digested and may have a range of effects on gastrointestinal processes. This group includes lactose, fructose in excess of glucose, fructans, and fructooligosaccharides (FOS, such as nystose and kestose), galacto-oligosaccharides (GOS such as raffinose and stachyose), and sugar polyols (sorbitol and mannitol) (Muir et al., 2009).

Fermentable oligosaccharides, disaccharides, monosaccharides and polyols are found in a wide variety of foods. Their dietary uptake mostly results from honey and fruits as watermelons, pears, and apples (fructose); milk and dairy products (lactose); rye, wheat, artichoke, garlic, onions, 
and broccoli (fructans and FOS); pulses (GOS); stone fruits and artificial sweeteners, mushrooms, broccoli, and cauliflower (sugar polyols) (Muir et al., 2009; Shepherd et al., 2013).

Fructans and FOS are the main FODMAPs in wheat-based products (Biesiekierski et al., 2011; Shewry and Hey, 2015; Ziegler et al., 2016). They are composed of fructose molecules linked to each other through fructosyl-fructose bonds and of one sucrose terminal end. Inulin-type and levan-type are linear fructans containing $\beta-(1 / 2)$ and $\beta-(6 / 2)$, respectively, fructosyl-fructose bonds. Both bonds are found in branched chain fructans called "graminans" (Verspreet et al., 2015). These bonds may be cleaved by several enzymes: endo-inulinase, exo- $\beta$-fructosidase, endo-levanase, $\beta$-fructofuranosidase (alias invertase), and levansucrase. Endo-inulinase and endo-levanase act on internal linkages, generating oligosaccharides with a lower degree of polymerization. Exo- $\beta$-fructosidase and $\beta$-fructofuranosidase liberate terminal fructose. Invertase, typically splitting sucrose into glucose and fructose, could act on fructans and FOS (Verspreet et al., 2013). Levansucrase generates sucrose from FOS and could further split sucrose into glucose and fructose (De Angelis et al., 2017). GOS are soluble, non-reducing $\alpha(1,6)$ galactosyl extensions of sucrose. From a chemical point of view, it would be more correct to name these FODMAPs as "Raffinose Family of Oligosaccharides," but they are usually referred to as "GOS." Elongation of the trisaccharide raffinose with galactose residues leads to stachyose and verbascose (Van Den Ende, 2013). $\alpha$-galactosidase acts on the $\alpha-1,6$ linkage, thus hydrolyzing GOS (Katrolia et al., 2014). In addition, inulinase, invertase, and levansucrase may cleave the bond between fructose and glucose in sucrose, raffinose, stachyose, and verbascose to yield glucose, melibiose, manninotriose, and manninotetraose, respectively. Thus, the combined action of $\alpha$-galactosidase and enzymes cleaving the fructose-glucose bond converts non-digestible GOS to carbohydrates with lower polymerization degree and potential prebiotic activity (Teixeira et al., 2012; Morel et al., 2015).

Fermentable oligosaccharides, disaccharides, monosaccharides and polyols' intake may be detrimental for health, being associated, for instance, with the onset of symptoms of irritable bowel syndrome (IBS) (Barrett, 2017). On the other hand, some FODMAPs are proven to be prebiotic, contributing to the healthy maintenance of intestinal microbiota (Muir et al., 2009; Halmos et al., 2014). Wheat-based products (e.g., bread, breakfast cereals, and pasta) account for a major part of daily consumed FODMAPs, due to their high consumption as staple foods worldwide (Verspreet et al., 2015). In particular, since ages of ancient civilizations (Babylonians, Egyptians, Greeks, and Romans), bread has been a major component of daily diet of several peoples (Chavan and Chavan, 2011). Volume increase of dough (flour and water) usually occurring during bread-making commonly relies on the use of biological leavening agents. Sourdough is the traditional leavening agent, resulting from the fermentation of cereal flour and water, by mainly lactic acid bacteria (LAB) and yeasts (De Vuyst et al., 2014; Gobbetti et al., 2014; Minervini et al., 2014).

Starting from the beginning of the twentieth century, sourdough was gradually replaced by baker's yeast (consisting of cell biomass mainly belonging to the yeast species Saccharomyces cerevisiae), a microbial starter culture produced at industrial level and distributed to bakers, which add it at low percentages (0.5$2.5 \%$ ) in bread dough to obtain leavening. The main consequence of this revolution was a decreased flavor, due to reduced fermentation times and almost exclusive yeast metabolic activities (Cauvain and Young, 2009). It has also been hypothesized that short fermentation may have contributed to bread intolerance through its effects on fermentation in the colon (Costabile et al., 2014). However, during the two last decades, an increasing number of consumers is demanding traditional bread endowed with higher aroma and taste and the main industrial reply consists in the rediscovery of sourdough fermentation (Decock and Cappelle, 2005; Cauvain and Young, 2009). This review focuses on the association between FODMAPs and IBS, beneficial effects of FODMAPs on healthy subjects and potential impact of biological leavening agents on FODMAPs content of bread.

\section{IBS, FODMAPS, AND DIET}

IBS is one of the most common type of functional bowel disorders, in which abdominal pain is associated with a change in bowel habits (Giorgio et al., 2015). Symptoms include diarrhea, constipation, bloating, distension, abdominal discomfort and/or pain and flatulence (Tuck et al., 2014; Marsh et al., 2016). The prevalence of IBS is estimated between 7 and $21 \%$ worldwide (Brandt et al., 2009; Lovell and Ford, 2012).

For some individuals the intake of FODMAPs may lead to an exacerbation of symptoms associated with IBS and other functional gut disorders (Gibson et al., 2015). The triggering of IBS symptoms by the consumption of FODMAPs is attributed to their slow or partial absorption in the small intestine. These carbohydrates are described as "fermentable" because they are likely to be fermented in the colon due to the absence, or reduced concentration, of hydrolase or because they are not absorbed completely (Staudacher et al., 2012). The gastrointestinal tract has no hydrolase capable of degrading fructans or GOS (Kolida and Gibson, 2007; Teixeira et al., 2012; Fedewa and Rao, 2014; Staudacher et al., 2014). Polyols are only partially digested and absorbed in the small intestine and reach the colon, where they are fermented by bacteria (Murillo et al., 2016). In the small intestine, free fructose is absorbed thanks to GLUT5 and GLUT2 transporters. GLUT5 is a facultative transporter specific for fructose, and provides carrier-mediated facilitated diffusion. Although present along the whole length of the small intestine, GLUT5 shows low capacity leading to slow uptake of luminal fructose. GLUT2 is a low-affinity, facultative transporter for glucose, fructose, and galactose, that is activated in presence of high luminal glucose concentrations. Although fructose absorption is markedly enhanced in presence of luminal free glucose, if the latter is present in excess, the risk of fructose malabsorption is greater, because of the low affinity characterizing GLUT2. Other factors have been shown to alter fructose absorption, but the underlying mechanisms have not been totally elucidated (Gibson et al., 2007).

Delivery of FODMAPs to the lumen of the distal small intestine and proximal large intestine may have adverse 


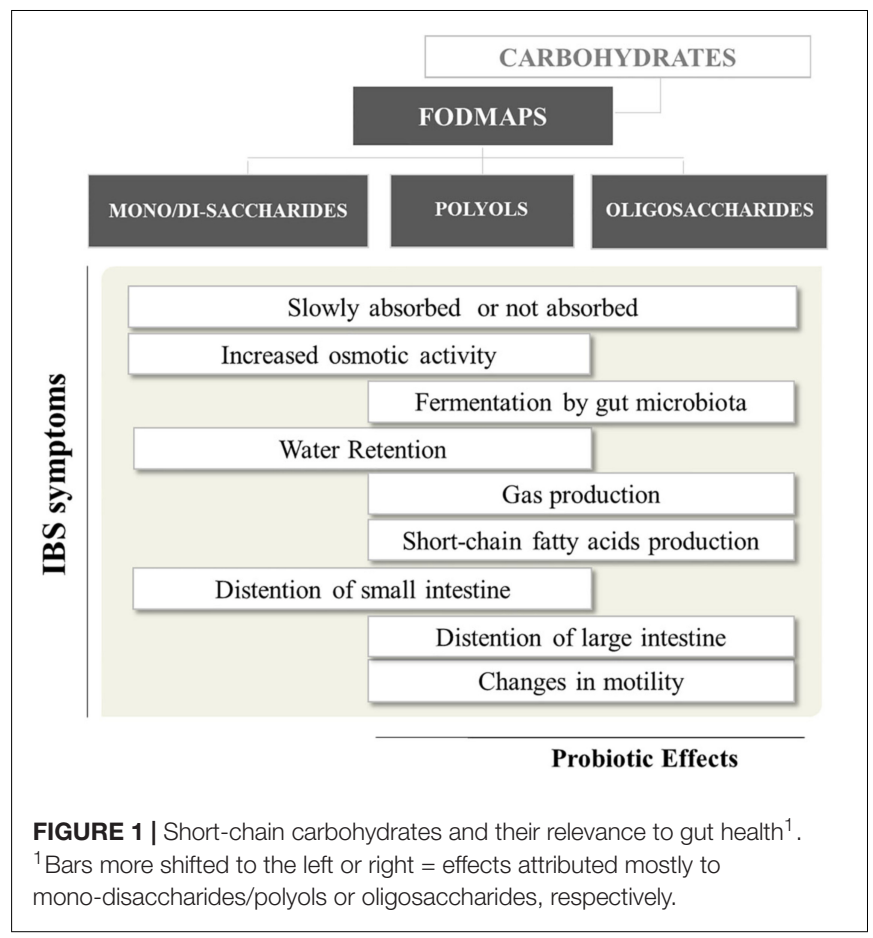

consequences in IBS patients (Figure 1). FODMAPs are believed to induce symptoms by two main mechanisms: (i) drawing water into the small intestine, causing distension, swelling, and discomfort; (ii) the rapid fermentation of FODMAPs would generates gas, distending the colon and causing flatulence, swelling and discomfort. The first mechanism is mostly related to low molecular mass FODMAPs (e.g., fructose), which are osmotically active in the intestinal lumen. Therefore, volume of fluid entering the bowel increases, and this may provide a natural laxative effect in healthy people, but may contribute to diarrhea in IBS sufferers. Regarding the second mechanism, intestinal bacteria rapidly ferment FODMAPs (e.g., FOS) releasing short-chain fatty acids (SCFA) and gases (e.g., carbon dioxide, hydrogen, and methane) (Giorgio et al., 2015). Hence, FODMAPs malabsorption and their fermentation in the proximal colon can add gas and water to the luminal contents, leading to distension and the onset of other symptoms (Ong et al., 2010). Moreover, patients with visceral hypersensitivity respond exaggeratedly to gas and fluid distension caused by malabsorption of carbohydrates (Major et al., 2017).

Although all FODMAP have similar physiological effects, each FODMAPs subgroup has a different gastrointestinal response, depending on molecular mass, rapidity of absorption and osmolarity (Murray et al., 2014). Overall, slowly absorbed fructose and polyols have a greater osmotic effect per molecule than fructans. Conversely, oligosaccharides, being scarcely absorbed across the small intestine, will have greater fermentative effects than fructose and polyols (Giorgio et al., 2015).

A limited number of effective treatment strategies is available for IBS (Tuck et al., 2014). Modulation of FODMAPs' intake in volume and type could be a means for controlling gut symptoms
(Gibson et al., 2015); that is why it is extremely necessary to know the concentration of FODMAPs present in the greatest possible number of foods. Control of FODMAPs ingested as food components is known as "low FODMAPs diet" (Marsh et al., 2016). The recommended total daily intake of FODMAPs in patients with IBS ranges from 5 to $30 \mathrm{~g}$ per day (Staudacher et al., 2012; Böhn et al., 2015). The reduction of gastrointestinal and systemic symptoms by restriction of FODMAPs ingestion has been observed by many research groups (Roest et al., 2013; Pedersen et al., 2014; Böhn et al., 2015; Barrett, 2017). The dietary manipulation of FODMAPs ingestion was able to impact on the total amount of gastrointestinal gas production and the spectrum of gas produced (hydrogen vs. methane) in healthy individuals and in patients with IBS (Ong et al., 2010). Patients subjected to diets containing low amount of FODMAPs present milder symptoms of IBS, through dietary restriction and rechallenges tests, used to determine individual tolerance to various short-chain carbohydrates (Halmos et al., 2014; Tuck et al., 2014).

The adoption of a low-FODMAPs diet presents some difficulties related to the lack of clear "cut-off levels" for FODMAPs content in foods and no available information on FODMAPs content on food packages. The definition of cut-off values should consider the amount of each particular FODMAPs present in a food, the typical serving size of food consumed in a single sitting, and the commonly triggered symptoms in individuals with IBS. To build a comprehensive composition database for FODMAPs in food, based on "ad hoc" studies, is essential for defining cut-off values (Muir et al., 2009). In addition, in long term, the low FODMAPs diet could change the gastrointestinal microbiota (Halmos et al., 2014; Tuck et al., 2014). Changes in total bacteria abundance, relative abundance of bifidobacteria (Staudacher et al., 2012) and strongly butyrate producing clostridial groups or Akkermansia muciniphila, positively associated with health, were described (Halmos et al., 2014). Moreover, the low FODMAPs diet could reduce the intestinal production of SCFA (Ong et al., 2010).

\section{BENEFICIAL EFFECTS OF FODMAP}

Fermentable oligosaccharides, disaccharides, monosaccharides and polyols are fermented in the colon to SCFA, which exert multiple beneficial effects on human health. Apart from being a major energy source for colonocytes, SCFA play a major immunological role in the gut, and help various physiological functions including colonic mobility and blood flow, and gastrointestinal $\mathrm{pH}$, which can influence uptake and absorption of electrolytes and nutrients (Den Besten et al., 2013; Tan et al., 2014). Furthermore, inulin-type fructans and GOS have been proposed for the treatment of metabolic endotoxaemia or low-grade inflammation in overweight/obese people (Morel et al., 2015; Fernandes et al., 2017). Some FODMAPs are being increasingly used in food industry as prebiotics, either as bioactive ingredients or as supplements, to promote colonic health. This kind of food/supplements should clearly be avoided by IBS patients. The higher FODMAPs' intake compared with that of the low FODMAPs or habitual diets was associated 
with specific stimulation of bacterial groups with putative health benefits (Halmos et al., 2014).

\section{HOW BREAD-MAKING MAY CHANGE FODMAPs LEVEL}

The FODMAPs content of breads depends on the nature of the grain-ingredient, as well as on the processing parameters (Biesiekierski et al., 2011). Despite the scarcity of papers that have quantified FODMAPs in breads, different concentrations of fructans $(0.1-1.7 \%$ of dry weight, $\mathrm{dw}$ ) have been reported in rye, spelt, and wheat breads. Fructose (0.1-2.3\% of dry weight), GOS (0.1-0.4\% of dw), FOS $(0.05-0.15 \%$ of $\mathrm{dw})$, sorbitol and mannitol (in traces) were also found (Biesiekierski et al., 2011; Whelan et al., 2011; Laatikainen et al., 2016). Besides that, pseudo-cereals and legumes are used for enriching/fortifying bread with nutritionally important components (e.g., minerals, vitamins, and phenolic compounds). This would increase the dietary intake of FODMAPs (especially GOS), because pseudocereals and legumes contain remarkable amounts of raffinose and stachyose (Muir et al., 2009; Curiel et al., 2015).

As stated in the introduction, bread accounts for a major part of daily consumed FODMAPs. Although the individual concentration of each subgroup of carbohydrates that comprises the FODMAPs may be low - except fructans, which are found in relatively high concentrations - consider the total content of all the subgroups of FODMAPs is more important than the individual levels of each subgroup, since the main effects of its intake can be summative. The fact that bread is one of the most important carbohydrate sources of the daily diet makes its quantity of FODMAPs relevant to IBS-sufferers.

Baker' yeast-leavened bread is typically obtained upon short fermentation (0.5-3 h), which causes a relatively limited hydrolysis of cereal components, including proteins and FODMAPs. On the opposite, sourdough biotechnology requires longer fermentation time. Sourdough is a complex microbial ecosystem, usually dominated by heterofermentative lactobacilli (e.g., Lactobacillus brevis, Lactobacillus fermentum, Lactobacillus plantarum, Lactobacillus rossiae, Lactobacillus reuteri, and Lactobacillus sanfranciscensis) and yeasts (e.g., S. cerevisiae, Kazachstania exigua, Kazachstania humilis, Torulaspora delbrueckii, Wickerhamomyces anomalus, and Pichia kudriavzevii). Microbial ecology of this ecosystem has been widely described in other reviews (De Vuyst et al., 2014; Gobbetti et al., 2016). Sourdough biotechnology may be exploited to improve flavor and texture, extend shelf-life and enhance nutritional and functional quality of leavened baked goods (Gobbetti et al., 2014). Most of these features are due to metabolic activities of LAB, especially on carbohydrates and proteins. During sourdough fermentation, the metabolism of carbohydrates depends on the available substrates, microbial and endogenous (flour) enzymes and interactions between microbial populations (e.g., competition and proto-cooperation). In detail, during typical (wheat and rye) sourdough fermentation, flour $\alpha$-amylase hydrolyzes starch to maltodextrins, which are then converted by flour $\beta$-amylase into maltose, the most abundant fermentable carbohydrate in dough. At dough stage, microbial invertase rapidly cleaves flour sucrose into glucose and fructose (Gänzle, 2014). Glucose is used as energy source, whereas fructose may be reduced by heterofermentative LAB to mannitol. Through reduction of fructose to mannitol, these bacteria convert acetyl-phosphate to acetate (instead of ethanol), thus gaining an extra mole of ATP (Gobbetti et al., 2005). Overall, all the fermentable carbohydrates (sucrose, maltose, glucose, and fructose) are quickly depleted during the first hours of fermentation, whereas carbohydrates with a higher degree of polymerization (such as fructans) are used later. This leads to hypothesize that long fermentation, such as that typically relying on sourdough, can provide a more pronounced degradation of FODMAPs. Contrarily to starch and fermentable carbohydrates, the mechanisms behind FODMAPs degradation have received less attention.

Among FODMAPs, fructans in wheat could be degraded upon sourdough fermentation. Indeed, few sourdough LAB, such as Lactobacillus amylovorus and Lactobacillus crispatus, are able to metabolize fructans (Loponen and Gänzle, 2018). Muller and Lier (1994) described that fructans are converted to fructose and sucrose, which are further metabolized into lactic acid by homofermentative LAB or into lactic acid, acetic acid, ethanol and $\mathrm{CO}_{2}$ by heterofermentative $\mathrm{LAB}$. In addition, reduction of fructose to mannitol, another FODMAP, frequently occurs in sourdough fermented by heterofermentative LAB (Gänzle, 2015). An extracellular, cell wall-associated $\beta$-fructosidase has been reported in strains of Lactobacillus paracasei as responsible for the degradation of fructans, leading to extracellular accumulation of sucrose and fructose (Makras et al., 2005; Goh et al., 2007).

Lactobacillus plantarum, L. fermentum, L. brevis, and Lactobacillus buchneri, isolated from vegetables, produce $\alpha$-galactosidase, acting on GOS (Silvestroni et al., 2002). In addition, gene coding for levansucrase was expressed by L. reuteri LTH5448, isolated from sourdough, more than 100 fold in presence of raffinose (Teixeira et al., 2012). This strain, also possessing $\alpha$-galactosidase activity, was used to ferment $\left(37^{\circ} \mathrm{C}, 24 \mathrm{~h}\right)$ faba bean flour, which was added to gluten-free bread dough containing, among other ingredients, baker's yeast and sorghum sourdough (previously fermented by the same strain). After $2 \mathrm{~h}$ of fermentation, GOS had been totally degraded. However, partial degradation of GOS to melibiose, manninotriose or mannino-tetraose was also observed when unfermented bean flour was used as ingredient, indicating that extracellular levansucrase rapidly acts on GOS, whereas subsequent intracellular $\alpha$-galactosidase activity proceeds slower. Comparable partial degradation of GOS was also observed in the control gluten-free dough, wherein L. reuteri LTH5448 had not been used (Teixeira et al., 2012). This could be explained by the action of yeast's invertase, which has a catalytic mechanism similar to levansucrase (Lammens et al., 2009). The invertase produced by $S$. cerevisiae is also able to degrade (partially) fructans to glucose and fructose. Indeed, during wheat whole meal bread-making, S. cerevisiae degraded almost $80 \%$ of the fructans initially present (Verspreet et al., 2013). Overall, yeasts may reduce the level of various FODMAPs during bread-making. For instance, Ziegler et al. (2016) showed that relatively long 
$(4.5 \mathrm{~h})$ baker's yeast fermentation results quite effective for reducing up to $90 \%$ the dough levels of fructans, raffinose, and excess of fructose, in five Triticum species. Struyf et al. (2017) developed an efficient yeast-based strategy, using an inulinase-secreting Kluyveromyces marxianus strain to reduce by more than $90 \%$ fructans levels in bread, upon $2.5 \mathrm{~h}$ of dough fermentation.

Based on the above-mentioned enzymatic activities, some sourdough lactobacilli (L. plantarum, L. fermentum, and L. brevis) could ferment GOS, FOS, and fructans (Saulnier et al., 2007; Pan et al., 2009; Kunová et al., 2011). In turn, when lactobacilli hydrolyze fructans, polyols and excess fructose are generated. Strains of L. plantarum and Lactobacillus curvatus isolated from rye sourdough were able to ferment fructose, mannitol, and sorbitol (Bartkiene et al., 2017). Wheat germ subjected to long fermentation with autochthonous strains of L. plantarum and L. rossiae showed 87 and $45 \%$ decrease of fructose and raffinose, respectively, compared to the initial concentrations (Rizzello et al., 2010). Similarly, the combined use of L. plantarum and L. brevis to ferment (at $30^{\circ} \mathrm{C}$, for $24 \mathrm{~h}$ ) pulses flour resulted in a decreased concentration of raffinose (of up to ca. 64\%), with respect to a control dough (obtained without bacterial inoculum) (Curiel et al., 2015).

Costabile et al. (2014) investigated the effects of breads, which differed in terms of fermentation time and biological leavening agent, on the colonic microbiota. In vitro batch culture experiments were run using feces from three subjects suffering from IBS and three healthy subjects. A significant increase in intestinal bifidobacterial populations, cultured in vitro, occurred after $8 \mathrm{~h}$ of fermentation with pre-digested sourdough breads. The same effect was not observed for breads produced with commercial yeast. Bifidobacteria do not produce gas and have been shown to benefit health of IBS patients (Clarke et al., 2012). $\delta$-Proteobacteria and most Gemmatimonadetes species decreased in both IBS and healthy subjects upon 24 h-long exposure to sourdough bread. In addition, in IBS subjects the latter produced significantly lower cumulative gas after $15 \mathrm{~h}$ of exposure, compared to baker's yeast breads. Overall this study suggests that sourdough bread may be beneficial for patients suffering from IBS, by influencing composition and metabolic profile of the human intestinal microbiota (Costabile et al., 2014). Another study has shown that low-FODMAPs rye breads, approximately $30 \%$ reduction of mannitol and fructans, caused less gastrointestinal symptoms, as abdominal pain, flatulence, stomach rum- and intestinal cramps (Laatikainen et al., 2016). A third study conducted by Pirkola et al. (2018) demonstrated that low-FODMAP rye bread leads to reduced colonic fermentation and flatulence severity when compared with regular rye bread consumption.

\section{FUTURE ASPECTS}

Future researches focusing on FODMAPs dietary contribution of bread will have to fill several knowledge gaps. First, information about FODMAPs content in food is limited, making difficult to understand their physiological importance (Muir et al., 2009;
Giorgio et al., 2015). With special regard to bread, FODMAPs concentration depends on intrinsic characteristics (e.g., cereal variety, growing locations, climate, and season), ingredients of bread recipes and processing methods. This gap is hard to be filled also because different methodologies of extraction and detection may lead to different concentrations. When detected through chromatography, some FODMAPs co-elute, obliging to perform a second chromatographic step with two mobile phases. In addition, some FODMAPs are present at concentrations very close to the limit of chromatographic detection, which may cause errors in quantification (Paramithiotis et al., 2007). Finally, detection of some FODMAPs (e.g., fructans) requires a combination of chromatography and enzymatic hydrolysis (Muir et al., 2009; Biesiekierski et al., 2011). Overall the finding of well-standardized methods of FODMAPs detection would be a key-point for food researchers, industries and consumers. Besides knowing FODMAPs concentrations, it is of utmost importance to elucidate the degradation pathways of the different FODMAPs subgroups and how they affect intestinal microbiota. This would help to evaluate the different symptomatic responses of subjects and to guide researchers in designing foods targeted for any specific group of consumers (Tuck et al., 2014).

As discussed in the previous paragraph, LAB and yeasts may degrade FODMAPs. Compared to baker's yeast, sourdough shows a great potential for driving FODMAPs concentrations of bread (and, overall, leavened baked goods), because it often includes both microbial populations. Therefore, future research should focus on how to combine enzyme activities of LAB and yeasts to reach the desired goal. In this context, given that some FODMAPs degrading enzymes are rather widespread in $\mathrm{LAB}$, one pillar is the selection of microbial strains possessing such enzymes and their use as sourdough starters.

Finally, the effect of FODMAPs degradation on bread quality should be evaluated. For instance, sucrose and fructose, generated upon partial hydrolysis of fructans, may be further used by bacteria to synthesize exopolysaccharides. These compounds protect bacteria against various environmental stresses, improve texture and delay bread staling (Ketabi et al., 2008; Dertli et al., 2016). In addition some exopolysaccharides have prebiotic activity (Ketabi et al., 2008; Torrieri et al., 2014). On the other hand, eventually, the EPS produced can be a fructosecomposed EPS and the degradation of this EPS during the dough preparation may again result in an increase in the fructose concentration, which is not favorable for IBS sufferers. Besides that, some $\mathrm{LAB}$ reduce fructose to mannitol, which would reduce the level of fructose but increase the other FODMAP. In this sense, the effects of sourdough fermentation on the content of FODMAPs will depend on the fermentation conditions and on which LAB species will be applied, offering a rather uncovered field to be clarified. As well as the possible increase in mannitol levels may affect the health of IBS-sufferers requires further studies.

Filling these knowledge gaps, it will be possible to modulate FODMAPs type and concentration in bread, allowing to reach two goals: (i) limiting the intake of FODMAPs in IBS patients to avoid undesirable gastrointestinal symptoms; (ii) attempting to increase their intake in healthy subjects. 


\section{AUTHOR CONTRIBUTIONS}

LM, FM, and PF researched and wrote the article. MS, FM, MG, and JDDL critically revised the article.

\section{REFERENCES}

Barrett, J. S. (2017). How to institute the low-FODMAP diet. J. Gastroenterol. Hepatol. 32, 8-10. doi: 10.1111/jgh.13686

Bartkiene, E., Bartkevics, V., Pugajeva, I., Krungleviciute, V., Mayrhofer, S., and Domig, K. (2017). The contribution of P. acidilactici, L. plantarum, and L. curvatus starters and L-(+)-lactic acid to the acrylamide content and quality parameters of mixed rye - Wheat bread. LWT Food Sci. Technol. 80, 43-50. doi: 10.1016/j.lwt.2017.02.005

Betts, J. A., and Gonzalez, J. T. (2016). Personalised nutrition: what makes you so special? Nutr. Bull. 41, 353-359. doi: 10.1111/nbu.12238

Biesiekierski, J. R., Rosella, O., Rose, R., Liels, K., Barrett, J. S., Shepherd, S. J., et al. (2011). Quantification of fructans, galacto-oligosacharides and other shortchain carbohydrates in processed grains and cereals. J. Hum. Nutr. Diet. 24, 154-176. doi: 10.1111/j.1365-277X.2010.01139

Böhn, L., Störsrud, S., Liljebo, T., Collin, L., Lindfors, P., Törnblom, H., et al. (2015). Diet low in FODMAP reduces symptoms of irritable bowel syndrome as well as traditional dietary advice: a randomized controlled trial. Gastroenterology 149, 1399-1407. doi: 10.1053/j.gastro.2015.07.054

Brandt, L. J., Chey, W. D., Foxx-Orenstein, A. E., Schiller, L. R., Schoenfeld, P. S., Spiegel, B. M., et al. (2009). An evidence-based position statement on the management of irritable bowel syndrome. Am. J. Gastroenterol. 104, 1-35. doi: 10.1038/ajg.2008.122

Cauvain, S. P., and Young, L. S. (2009). Tecnologia da Panificação, 2nd Edn. São Paulo: Editora Manole, 418.

Chavan, R. S., and Chavan, S. R. (2011). Sourdough technology-a traditional way for wholesome foods: a review. Compr. Rev. Food Sci. Food Saf. 10, 169-182. doi: 10.1111/j.1541-4337.2011.00148.x

Clarke, G., Cryan, J. F., Dinan, T. G., and Quigley, E. M. (2012). Review article: probiotics for the treatment of irritable bowel syndrome-focus on lactic acid bacteria. Aliment. Pharmacol. Ther. 35, 403-413. doi: 10.1111/j.1365-2036. 2011

Costabile, A., Santarelli, S., Claus, S. P., Sanderson, J., Hudspith, B. N., Brostoff, J., et al. (2014). Effect of breadmaking process on in vitro gut microbiota parameters in irritable bowel syndrome. PLoS One 9:e111225. doi: 10.1371/ journal.pone. 0111225

Curiel, J. A., Coda, R., Centomani, I., Summo, C., Gobbetti, M., and Rizzello, C. G. (2015). Exploitation of the nutritional and functional characteristics of traditional Italian legumes: the potential of sourdough fermentation. Int. J. Food Microbiol. 196, 51-61. doi: 10.1016/j.ijfoodmicro.2014.11.032

De Angelis, M., Garruti, G., Minervini, F., Bonfrate, L., Portincasa, P., and Gobbetti, M. (2017). The food-gut human axis: the effects of diet on gut microbiota and metabolome. Curr. Med. Chem. doi: 10.2174/ 0929867324666170428103848 [Epub ahead of print].

De Toro-Martín, J., Arsenault, B. J., Després, J. P., and Vohl, M. C. (2017). Precision nutrition: a review of personalized nutritional approaches for the prevention and management of metabolic syndrome. Nutrients 9:E913. doi: $10.3390 /$ nu 9080913

De Vuyst, L., Van Kerrebroeck, S., Harth, H., Huys, G., Daniel, H.-M., and Weckx, S. (2014). Microbial ecology of sourdough fermentations: diverse or uniform? Food Microbiol. 37, 11-29. doi: 10.1016/j.fm.2013.06.002

Decock, P., and Cappelle, S. (2005). Bread technology and sourdough technology. Trends Food Sci. Technol. 16, 113-120. doi: 10.1016/j.tifs.2004.04.012

Den Besten, G., Van Eunen, K., Groen, A. K., Venema, K., Reijngoud, D. J., and Bakker, B. M. (2013). The role of short-chain fatty acids in the interplay between diet, gut microbiota, and host energy metabolism. J. Lipid Res. 54, 2325-2340. doi: $10.1194 /$ jlr.R036012

Dertli, E., Mercan, E., Arı cl, M., Y Yllmaz, M. T., and Sağd $\iota c ̧$, O. (2016). Characterization of lactic acid bacteria from Turkish sourdough and determination of their exopolysaccharide (EPS) production characteristics. LWT Food Sci. Technol. 71, 116-124. doi: 10.1016/j.lwt.2016.03.030

\section{ACKNOWLEDGMENTS}

This research did not receive any specific grant from funding agencies in the public, commercial, or not-for-profit sectors.

Fedewa, A., and Rao, S. S. C. (2014). Dietary fructose intolerance, fructan intolerance and FODMAPs. Curr. Gastroenterol. Rep. 16:370. doi: 10.1007/ s11894-013-0370-0

Fernandes, R., Do Rosario, V. A., Mocellin, M. C., Kuntz, M. G., and Trindade, E. B. (2017). Effects of inulin-type fructans, galacto-oligosaccharides and related synbiotics on inflammatory markers in adult patients with overweight or obesity: a systematic review. Clin. Nutr. 36, 1197-1206. doi: 10.1016/j.clnu.2016. 10.003

Gänzle, M. G. (2014). Enzymatic and bacterial conversions during sourdough fermentation. Food Microbiol. 37, 2-10. doi: 10.1016/j.fm.2013.04.007

Gänzle, M. G. (2015). Lactic metabolism revisited: metabolism of lactic acid bacteria in food fermentations and food biotechnology. Curr. Opin. Food Sci. 2, 106-117. doi: 10.1016/j.cofs.2015.03.001

Gibson, P. R., Newnham, E., Barrett, J. S., Shepherd, S. J., and Muir, J. G. (2007). Fructose malabsorption and the bigger picture. Aliment. Pharmacol. Ther. 25, 349-363. doi: 10.1111/j.1365-2036.2006.03186.x

Gibson, P. R., Varney, J., Malakar, S., and Muir, J. G. (2015). Food components and irritable bowel syndrome. Gastroenterology 148, 1158-1174. doi: 10.1053/j. gastro.2015.02.005

Giorgio, R., Volta, U., and Gibson, P. R. (2015). Sensitivity to wheat, gluten and FODMAP in IBS: facts or fiction? Gut 65, 169-178. doi: 10.1136/gutjnl-2015309757

Gobbetti, M., De Angelis, M., Corsetti, A., and Di Cagno, R. (2005). Biochemistry and physiology of sourdough lactic acid bacteria. Trends Food Sci. Technol. 16, 57-69. doi: 10.1016/j.tifs.2004.02.013

Gobbetti, M., Minervini, F., Pontonio, E., Di Cagno, R., and De Angelis, M. (2016). Drivers for the establishment and composition of the sourdough lactic acid bacteria biota. Int. J. Food Microbiol. 239, 3-18. doi: 10.1016/j.ijfoodmicro.2016. 05.022

Gobbetti, M., Rizzello, C. G., Di Cagno, R., and De Angelis, M. (2014). How the sourdough may affect the functional features of leavened baked goods. Food Microbiol. 37, 30-40. doi: 10.1016/j.fm.2013.04.012

Goh, Y. J., Lee, J. H., and Hutkins, R. W. (2007). Functional analysis of the fructooligosaccharide utilization operon in Lactobacillus paracasei 1195. Appl. Environ. Microbiol. 73, 5716-5724. doi: 10.1128/AEM.00805-07

Halmos, E. P., Christophersen, C. T., Bird, A. R., Shepherd, S. J., Gibson, P. R., and Muir, J. G. (2014). Diets that differ in their FODMAP content alter the colonic luminal microenvironment. Gut 64, 93-100. doi: 10.1136/gutjnl-2014307264

Katrolia, P., Rajashekhara, E., Yan, Q., and Jiang, Z. (2014). Biotechnological potential of microbial $\alpha$-galactosidases. Crit. Rev. Biotechnol. 34, 307-317. doi: 10.3109/07388551.2013.794124

Ketabi, A., Soleimanian-Zad, S., Kadivar, M., and Sheikh-Zeinoddin, M. (2008). Production of microbial exopolysaccharides in the sourdough and its effects on the rheological properties of dough. Food Res. Int. 41, 948-951. doi: 10.1016/j. foodres.2008.07.009

Kolida, S., and Gibson, P. R. (2007). Prebiotic capacity of inulin-type fructans. J. Nutr. 137, 2503-2506. doi: 10.1093/jn/137.11.2503S

Kunová, G., Rada, V., Lisová, I., Rocková, S., and Vlková, E. (2011). In vitro fermentability of prebiotic oligosaccharides by lactobacilli. Czech J. Food Sci. 29, 49-54. doi: 10.17221/306/2011-CJFS

Laatikainen, R., Koskenpato, J., Hongisto, S. M., Loponen, J., Poussa, T., Hillilä, M., et al. (2016). Randomised clinical trial: low-FODMAP rye bread vs. regular rye bread to relieve the symptoms of irritable bowel syndrome. Aliment. Pharmacol. Ther. 44, 460-470. doi: 10.1111/apt.13726

Lammens, W., Le Roy, K., Schroeven, L., Van Laere, A., Rabijns, A., and Van Den Ende, W. (2009). Structural insights into glycoside hydrolase family 32 and 68 enzymes: functional implications. J. Exp. Bot. 60, 727-740. doi: 10.1093/jxb/ ern333

Loponen, J., and Gänzle, M. G. (2018). Use of sourdough in low FODMAP baking. Foods 7:E96. doi: 10.3390/foods7070096 
Lovell, R. M., and Ford, A. C. (2012). Global prevalence of and risk factors for irritable bowel syndrome: a meta-analysis. Clin. Gastroenterol. Hepatol. 10, 712-721. doi: 10.1016/j.cgh.2012.02.029

Major, G., Pritchard, S., Murray, K., Alappadan, J. P., Hoad, C. L., Marciani, L., et al. (2017). Colon hypersensitivity to distension, rather than excessive gas production, produces carbohydrate-related symptoms in individuals with irritable bowel syndrome. Gastroenterology 152, 124-133. doi: 10.1053/j.gastro. 2016.09.062

Makras, L., Van Acker, G., and De Vuyst, L. (2005). Lactobacillus paracasei subsp. paracasei 8700:2 degrades inulin-type fructans exhibiting different degrees of polymerization. Appl. Environ. Microbiol. 71, 6531-6537. doi: 10.1128/AEM.71. 11.6531-6537.2005

Marsh, A., Eslick, E. M., and Eslick, G. D. (2016). Does a diet low in FODMAP reduce symptoms associated with functional gastrointestinal disorders? A comprehensive systematic review and meta-analysis. Eur. J. Nutr. 55, 897-906. doi: 10.1007/s00394-015-0922-1

Minervini, F., De Angelis, M., Di Cagno, R., and Gobbetti, M. (2014). Ecological parameters influencing microbial diversity and stability of traditional sourdough. Int. J. Food Microbiol. 171, 136-146. doi: 10.1016/j.ijfoodmicro. 2013.11.021

Morel, F. B., Dai, Q., Ni, J., Thomas, D., Parnet, P., and Fança-Berthon, P. (2015). $\alpha$-Galacto-oligosaccharides dose-dependently reduce appetite and decrease inflammation in overweight adults. J. Nutr. 145, 2052-2059. doi: 10.3945/jn. 114.204909

Muir, J. G., Rose, R., Rosella, O., Liels, K., Barrett, J. S., Shepherd, S. J., et al. (2009). Measurement of short-chain carbohydrates in common Australian vegetables and fruits by high-performance liquid chromatography (HPLC). J. Agric. Food Chem. 57, 554-565. doi: 10.1021/jf802700e

Muller, M., and Lier, D. (1994). Fermentation of fructans by epiphytic lacticacid bacteria. J. Appl. Bacteriol. 76, 406-411. doi: 10.1111/j.1365-2672.1994.tb 01647.x

Murillo, Z., Arévalo, E., and Jáuregui, P. (2016). Diet low in fermentable oligosaccharides, disaccharides, monosaccharides and polyols (FODMAPs) in the treatment of irritable bowel syndrome: indications and design. Endocrinol. Nutr. 63, 132-138. doi: 10.1016/j.endonu.2015.10.009

Murray, K., Wilkinson-Smith, V., Hoad, C., Costigan, C., Cox, E., Lam, C., et al. (2014). Differential effects of FODMAP (Fermentable Oligo-, Di-, MonoSaccharides and Polyols) on small and large intestinal contents in healthy subjects shown by MRI. Am. J. Gastroenterol. 109, 110-119. doi: 10.1038/ajg. 2013.386

Ong, D. K., Mitchell, S. B., Barrett, J. S., Shepherd, S. J., Irving, P. M., Biesiekierski, J. R., et al. (2010). Manipulation of dietary short chain carbohydrates alters the pattern of gas production and genesis of symptoms in irritable bowel syndrome. J. Gastroenterol. Hepatol. 25, 1366-1373. doi: 10.1111/j.1440-1746.2010.06370.x

Pan, X., Wu, T., Zhang, L., Cai, L., and Song, Z. (2009). Influence of oligosaccharides on the growth and tolerance capacity of lactobacilli to simulated stress environment. Lett. Appl. Microbiol. 48, 362-367. doi: 10.1111/ j.1472-765X.2008.02539.x

Paramithiotis, S., Sofou, A., Tsakalidou, E., and Kalantzopoulos, G. (2007). Flour carbohydrate catabolism and metabolite production by sourdough lactic acid bacteria. World J. Microbiol. Biotechnol. 23, 1417-1423. doi: 10.1007/s11274007-9384-9

Pedersen, N., Andersen, N. N., Végh, Z., Jensen, L., Ankersen, D. V., Felding, M., et al. (2014). Ehealth: low FODMAP diet vs Lactobacillus rhamnosus GG in irritable bowel syndrome. World J. Gastroenterol. 20, 16215-16226. doi: 10. 3748/wjg.v20.i43.16215

Pirkola, L., Laatikainen, R., Loponen, J., Hongisto, S. M., Hillilä, M., Nuora, A., et al. (2018). Low-FODMAP vs regular rye bread in irritable bowel syndrome: randomized SmartPill ${ }^{\circledR}$ study. World J. Gastroenterol. 24, 1259-1268. doi: 10.3748/wjg.v24.i11.1259

Rizzello, C. G., Nionelli, L., Coda, R., De Angelis, M., and Gobbetti, M. (2010). Effect of sourdough fermentation on stabilization, and chemical and nutritional characteristics of wheat germ. Food Chem. 119, 1079-1089. doi: 10.1016/j. foodchem.2009.08.016

Roest, R. H., Dobbs, B. R., Chapman, B. A., Batman, B., O’Brien, L. A., Leeper, J. A., et al. (2013). The low FODMAP diet improves gastrointestinal symptoms in patients with irritable bowel syndrome: a prospective study. Int. J. Clin. Pract. 67, 895-903. doi: 10.1111/ijcp.12128
Saulnier, D. M. A., Molenaar, D., De Vos, W. M., Gibson, G. R., and Kolida, S. (2007). Identification of prebiotic fructooligosaccharide metabolism in Lactobacillus plantarum WCFS1 through microarrays. Appl. Environ. Microbiol. 73, 1753-1765. doi: 10.1128/AEM.01151-06

Shepherd, S. J., Lomer, M. C., and Gibson, P. R. (2013). Short-chain carbohydrates and functional gastrointestinal disorders. Am. J. Gastroenterol. 108, 707-717. doi: 10.1038 /ajg. 2013.96

Shewry, P. R., and Hey, S. J. (2015). The contribution of wheat to human diet and health. Food Energy Secur. 4, 178-202. doi: 10.1002/fes3.64

Silvestroni, A., Connes, C., Sesma, F., de Giori, G. S., and Piard, J.-C. (2002). Characterization of the mela locus for $\alpha$-galactosidase in Lactobacillus plantarum. Appl. Environ. Microbiol. 68, 5464-5471. doi: 10.1128/AEM.68.11. 5464-5471.2002

Staudacher, H. M., Irving, P. M., Lomer, M. C. E., and Whelan, K. (2014). Mechanisms and efficacy of dietary FODMAP restriction in IBS. Nat. Rev. Gastroenterol. Hepatol. 11, 256-266. doi: 10.1038/nrgastro.2013.259

Staudacher, H. M., Lomer, M. C. E., Anderson, J. L., Barrett, J. S., Muir, J. G., Irving, P. M., et al. (2012). Fermentable carbohydrate restriction reduces luminal Bifidobacteria and gastrointestinal symptoms in patients with irritable bowel syndrome. J. Nutr. 142, 1510-1518. doi: 10.3945/jn.112.159285

Struyf, N., Laurent, J., Lefevere, B., Verspreet, J., Verstrepen, K. J., and Courtin, C. M. (2017). Establishing the relative importance of damaged starch and fructan as sources of fermentable sugars in wheat flour and whole meal bread dough fermentations. Food Chem. 218, 89-98. doi: 10.1016/j.foodchem.2016. 09.004

Tan, J., McKenzie, C., Potamitis, M., Thorburn, A. N., Mackay, C. R., and Macia, L. (2014). The role of short-chain fatty acids in health and disease. Adv. Immunol. 121, 91-119. doi: 10.1016/B978-0-12-800100-4.00003-9

Teixeira, J. S., Mcneill, V., and Gänzle, M. G. (2012). Levansucrase and sucrose phoshorylase contribute to raffinose, stachyose, and verbascose metabolism by lactobacilli. Food Microbiol. 31, 278-284. doi: 10.1016/j.fm.2012.03.003

Torrieri, E., Pepe, O., Ventorino, V., Masi, P., and Cavella, S. (2014). Effect of sourdough at different concentrations on quality and shelf life of bread. LWT Food Sci. Technol. 56, 508-516. doi: 10.1016/j.lwt.2013.12.005

Tuck, J., Muir, J. G., Barrett, J. S., and Gibson, P. R. (2014). Fermentable oligosaccharides, disaccharides, monosaccharides and polyols: role in irritable bowel syndrome. Expert Rev. Gastroenterol. Hepatol. 8, 819-834. doi: 10.1586/ 17474124.2014.917956

Van Den Ende, W. (2013). Multifunctional fructans and raffinose family oligosaccharides. Front. Plant Sci. 4:247. doi: 10.3389/fpls.2013.00247

Verspreet, J., Dornez, E., Van den Ende, W., Delcour, J. A., and Courtin, C. M. (2015). Cereal grain fructans: structure, variability and potential health effects. Trends Food Sci. Technol. 43, 32-42. doi: 10.1016/j.tifs.2015.01.006

Verspreet, J., Hemdane, S., Dornez, E., Cuyvers, S., Delcour, J. A., and Courtin, C. M. (2013). Maximizing the concentrations of wheat grain fructans in bread by exploring strategies to prevent their yeast (Saccharomyces cerevisiae) - mediated degradation. J. Agric. Food Chem. 61, 1397-1404. doi: 10.1021/ jf3050846

Whelan, K., Abrahmsohn, O., David, G. J., Staudacher, H., Irving, P., Lomer, M. C., et al. (2011). Fructan content of commonly consumed wheat, rye and glutenfree breads. Int. J. Food Sci. Nutr. 62, 498-503. doi: 10.3109/096374866.2011. 553588

Ziegler, J. U., Steinera, D., Longin, C. F. H., Würschum, T., Schweiggert, R. M., Carle, R., et al. (2016). Wheat and the irritable bowel syndrome - FODMAP levels of modern and ancient species and their retention during bread making. J. Funct. Foods 25, 257-266. doi: 10.1016/j.jff.2016.05.019

Conflict of Interest Statement: The authors declare that the research was conducted in the absence of any commercial or financial relationships that could be construed as a potential conflict of interest.

Copyright (c) 2018 Menezes, Minervini, Filannino, Sardaro, Gatti and De Dea Lindner. This is an open-access article distributed under the terms of the Creative Commons Attribution License (CC BY). The use, distribution or reproduction in other forums is permitted, provided the original author(s) and the copyright owner(s) are credited and that the original publication in this journal is cited, in accordance with accepted academic practice. No use, distribution or reproduction is permitted which does not comply with these terms. 